\title{
sur la gestion et les finances publiques En Mars 2021
}

\section{BUDGET DE L'ÉTAT ET DES OPÉRATEURS}

\section{$\lambda$ Données générales sur les finances publiques}

Projections macroéconomiques 2021 2023

Dans une publication du 16 mars 2021, la Banque de France a présenté ses projections macroéconomiques pour la période 2021-2023.

La Banque de France prévoit un rebond de l'activité économique à partir du second semestre 2021, dès lors que la progression de la vaccination permettrait la levée progressive des restrictions sanitaires, entraînant un rebond de la consommation des ménages. Selon la Banque de France, sur l'ensemble de l'année 2021, le PIB pourrait progresser de 5,5\%. Le rebond se prolongerait en 2022, avec une croissance toujours très vigoureuse, d'environ $4 \%$. En 2023, la croissance reviendrait à un niveau plus commun, autour de $2 \%$. Le rebond attendu de la consommation des ménages apparaît d'autant plus probable que leur revenu a été globalement préservé pendant la crise, grâce, à la fois, aux amortisseurs publics et à une résistance notable du marché du travail. Le chômage resterait significativement inférieur à $10 \%$ au cours de la période 2021-2023. L'inflation serait assez volatile en 2021, évoluant en glissement annuel entre $0,5 \%$ et $1,5 \%$, mais resterait très modérée sur l'ensemble de l'horizon de projection, autour de $1 \%$, en moyenne. La Banque de France souligne cependant que les aléas pouvant affecter cette projection restent importants en raison notamment des fortes incertitudes sanitaires.

Rapport de la Commission pour l'avenir des finances publiques

La Commission pour l'avenir des finances publiques, présidée par Jean Arthuis, a été mis- sionnée le 4 décembre 2020 par le Premier ministre, dans le contexte d'une forte hausse de la dette publique liée au Covid-19. Elle a rendu ses travaux le 18 mars dans un rapport intitulé "Nos finances publiques post-Covid-19: pour de nouvelles règles du jeu».

Le rapport met en évidence que, si la France poursuit le même rythme d'augmentation des dépenses publiques, l'endettement poursuivra sa hausse continue dans les prochaines années et cela dans la plupart des scénarios plausibles sur la croissance économique. Sans perspective de stabilisation, la situation apparaîtrait alors comme intenable à long terme. Si la crise a mis en avant le rôle décisif de l'intervention publique, elle devrait être aussi une opportunité pour imaginer et mettre en œuvre, à moyen terme, une nouvelle stratégie pour les finances publiques. Pour la Commission, cette nouvelle stratégie doit tout d'abord éviter de reproduire les erreurs du passé.

Compte tenu du niveau déjà élevé des impôts, la Commission considère qu'il n'est pas raisonnable d'envisager de faire reposer la maîtrise des finances publiques sur la perspective d'augmentation des prélèvements obligatoires. La priorité devrait donc aller à la maîtrise des dépenses, de faire en sorte qu'elles progressent moins vite que les recettes. Cette maîtrise des dépenses devrait s'inscrire dans la durée : la soutenabilité des finances publiques doit reposer sur la capacité à maintenir dans le temps une dynamique des dépenses en deçà de celles des recettes. Le rapport précise que la transformation radicale de la gouvernance applicable aux finances publiques est une condition de la réussite de la stratégie d'avenir qui repose sur 3 principes : créer une norme en dépense pluriannuelle et en faire une nouvelle boussole des finances publiques, installer une vraie vigie budgétaire pour une approche de long terme, approfondir le débat parlementaire pour une 
large appropriation démocratique des enjeux de finances publiques.

\section{त Patrimoine de l'État}

Bilan des dons mobiliers de l'État aux associations

Dans un communiqué de presse du 12 mars 2021, la Direction générale des finances publiques a dressé un bilan des dons mobiliers de l'État aux associations: près de 3000 biens ont déjà été distribués depuis le lancement en octobre dernier du site internet https://dons. encheres-domaine.gouv.fr/. Ce site permet aux administrations de donner des biens mobiliers d'occasion dont elles n'ont plus l'usage. La gamme des biens est très large avec comme principaux biens du mobilier de bureau et du matériel informatique mais d'autres biens plus insolites peuvent également être proposés (ex. matériel de scénographie, scientifique, vêtements).

Depuis la dernière loi de finances, les biens sont d'abord proposés à d'autres services de I'État et à des établissements ou des collectivités locales et sont ensuite proposés à des associations reconnues d'utilité publique mais aussi à des fondations. C'est ainsi que des dons ont été faits, entre autres, à ATD quart Monde, à la Croix rouge, au Secours populaire ou aux Restaurants du cœur. Cette démarche impulsée par les services du Domaine, qui relèvent de la DGFIP, permet à la fois de contribuer à l'économie circulaire et au développement solidaire.

\section{Budget des opérateurs}

La Banque de France présente ses résultats et son rapport annuel

Le 23 mars 2021, la Banque de France a publié ses résultats et son rapport annuel pour l'année 2020. Le résultat ordinaire avant impôt s'établit à 4,97 Mds€, contre 6,49 Mds l'an dernier. Comme chaque année, une grande partie du résultat de la Banque de France est reversée à l'État, et donc à la collectivité, sous forme d'impôt sur les bénéfices et de dividendes. Le montant versé cette année atteint un montant total de 4,24 Mds€. Le produit net des activités de la Banque de France s'établit à 6,4 Mds€ en 2020, en baisse de $25 \%$ par rapport à 2019. Cette baisse est liée essentiellement à la politique monétaire : augmentation des charges liées aux opérations de refinancement et baisse des revenus tirés des titres détenus. Au passif, on observe une forte hausse des dépôts des établissements de crédit en contrepartie, tandis que l'encours des billets en euros en circulation de la Banque de France continue de croître (+27 Mds€ d'une fin d'année sur l'autre, soit $+11 \%)$.

La Banque de France indique avoir élaboré son prochain plan stratégique "Construire ensemble $2024 »$. Amorcé de manière participative dès janvier 2020, il intègre les enseignements de la crise sanitaire et répond aux défis du sujet environnemental. Pour cela, la Banque de France a retenu 30 actions autour de 4 objectifs : par l'expertise et l'indépendance, soutenir l'économie ; par l'ouverture, servir la société et l'Europe ; par la performance durable, assurer I'avenir ; par la solidarité, être une entreprise attractive. Dix repères de résultats jugeront $d u$ succès de ce plan, dans le cadre de sa gestion efficace des moyens.

\section{La Grande École du numérique}

Dans un référé du 22 décembre 2020, publié le 8 mars 2021 sur le site de la Cour des comptes, cette dernière dresse un bilan de la mise en œuvre des missions de la Grande École du numérique.

Groupement d'intérêt public (GIP) constitué en 2016 entre l'État, Pôle emploi, I'association Régions de France, divers acteurs de l'emploi et de la formation professionnelle, ainsi que des entreprises privées, la Grande École du numérique, dont la mission principale est de labelliser et de financer des formations, permet à des publics éloignés de l'emploi d'accéder à une formation aux métiers du numérique. Cet objectif correspond à un vrai besoin, tant pour l'économie qu'en matière de soutien à l'emploi. En trois ans, le GIP a ainsi financé la formation de près de 28000 stagiaires, en majorité peu ou pas diplômés, pour un montant total d'environ $48 \mathrm{M€}$.

Selon la Cour, les missions de la Grande École du numérique souffrent toutefois de lacunes et d'irrégularités, notamment d'un système de conventionnement critiquable. Les modalités de financement des organismes de formations retenues par l'administration, puis par le GIP après sa création, s'écartent de la règle de droit et s'avèrent peu protectrices des deniers publics. Aussi, l'usage systématique de la subvention, au détriment des procédures de la commande publique, soulève des interrogations au regard des normes applicables. Plus globalement, le système de financement des formations ne paraît pas respecter le droit de I'Union européenne. 
Au demeurant, la Cour estime que la gouvernance de l'École est à réformer et son suivi par I'État à renforcer. La Cour formule dix recommandations au total.

\section{COMPTABILITÉ PUBLIQUE}

\section{$\pi$ Règlementation comptable}

Olivier Dussopt revient sur quelques propositions du rapport Bassères

Dans un entretien accordé à Bastien Scordia pour Acteurs Publics, publié le 22 mars 2021, le ministre chargé des comptes publics Olivier Dussopt revient sur les propositions du rapport de Jean Bassères relatif à la responsabilisation des gestionnaires publics.

Pour le ministre, les contrôles actuels, bien que nécessaires en matière de gestion budgétaire, sont très formels et ne permettent pas la fluidité des actes de gestion. Sans les supprimer totalement, Olivier Dussopt considère nécessaire de les adapter pour les rendre plus efficaces et en adéquation avec la responsabilité des gestionnaires. Pour lui, «c'est une réflexion technique qui accompagne des métiers de back office extrêmement précieux pour la mise en œuvre de nos politiques publiques $»$.

Interrogé sur quelques propositions emblématiques du rapport Bassères, le ministre a indiqué que la nature juridictionnelle de la Cour des comptes ne sera pas modifiée et que le principe de séparation ordonnateur-comptable ne sera pas supprimé.

Olivier Dussopt indique vouloir remettre d'ici à la fin de l'année au Premier ministre ses propositions de réforme de la responsabilité des gestionnaires publics. Dans cette attente, il espère trouver un consensus sur le sujet: «En tout état de cause, le nouveau régime de responsabilité des gestionnaires publics ne pourra être que le fruit d'un travail partagé entre l'administration, la Cour des comptes, le Conseil d'État et les représentants des collectivités territoriales ». Le ministre souhaite ensuite pouvoir rapidement saisir le Parlement en reconnaissant toutefois que « le calendrier parlementaire ne nous laisse que peu d'opportunités».

\section{FINANCES LOCALES}

\section{$\lambda$ Étude de la DGCL sur les dépenses des régions}

Avant-projet de loi dit «4D » relatif à la différenciation, la décentralisation, la déconcentration et portant diverses mesures de simplification de l'action publique locale

Ce texte de loi, dont on pensait qu'il allait finir aux oubliettes, semble malgré tout faire son bout de chemin, avec quelques dispositions intéressantes en matière financière. Ainsi, en matière de lutte contre le réchauffement climatique et préservation de la biodiversité, l'article 11 du projet de loi prévoit un transfert de l'Agence de l'environnement vers les régions de «l'attribution de subventions et de concours financiers en matière de transition énergétique et d'économie circulaire. Elle conclut à cette fin une convention de transition énergétique régionale qui définit le montant du financement alloué à la région ainsi que les objectifs à atteindre ». Ce projet de loi envisage, par ailleurs, une expérimentation en matière de recentralisation du RSA, avec en contrepartie des engagements des conseils départementaux sur les politiques d'insertion. L'article 24 ouvre également, à contre-courant de la spécialisation des compétences, la possibilité pour les communes, $\mathrm{EPCl}$, départements et régions de participer au financement du programme d'investissement des établissements de santé publics et privés.

Note d'information relative aux informations fiscales utiles à la préparation des budgets primitifs locaux pour 2021

Tous les ans, Bercy publie une note d'information sur les évolutions juridiques de la loi de finances pour 2021 et la dernière loi de finances rectificative pour 2020. Cette note retrace en premier lieu les évolutions à venir en matière de réforme de la taxe d'habitation et des impôts de production. Puis, et c'est surtout là son intérêt, elle détaille les mesures votées récemment. Ainsi, elle revient sur :

- la détermination des valeurs locatives notamment en raison du fait que l'article 132 de la loi de finances pour 2021 exclut de la méthode dite « comptable » d'évaluation de la valeur locative, les installations de stockage des déchets non dangereux ;

- les exonérations et notamment sur celles s'appliquant dès 2021 comme dans les zones de revitalisation de centres-villes (ZRCV) ou les zones de revitalisation des commerces en milieu rural (ZoRCoMiR) ;

- la suppression des taxes dites «funéraires";

- la garantie de recettes exceptionnelle du bloc communal, avec un mécanisme de compensation reconduit partiellement en 2021. 
Commission pour l'avenir des finances publiques

La Commission dite Arthuis chargée de réfléchir sur la dette Covid aborde la question des collectivités territoriales. En premier lieu, ce rapport prend le parti de s'interroger sur le nombre élevé de collectivités. Ce document rappelle ainsi que la France compte 93700 entités dans les administrations locales en France contre 15000 en Allemagne. Puis, il livre sa logique globale, qui repose sur la création d'une norme en dépense pluriannuelle. Dans le même temps, ce rapport souhaite réactualiser la contractualisation et surtout la généraliser à l'ensemble des collectivités et groupements et également étendre son champ d'application aux budgets annexes. Enfin, ce rapport travaille à la mise en place d'une conférence nationale des finances publiques, associant les collectivités locales, les partenaires sociaux et les citoyens.

Regard financier sur les communes de métropole jusqu'à 3500 habitants

La Banque postale livre son rapport sur les communes de moins de 3500 habitants pour la troisième année de suite. Au 1er janvier 2019, la France comptait 31746 communes de moins de 3500 habitants. Ces communes ont géré, en 2019, un total de 22,6 Mds€, dont 7,5 Mds€ affectés à l'investissement local (hors remboursement de la dette). L'encours de dette de ces communes s'avère finalement assez limité, représentant 13,6 Mds€ soit $24,3 \%$ de l'encours total, soit un montant de $640 €$ par habitant. Cependant, suivant les strates démographiques, les écarts sont assez variables. La Banque postale propose ainsi un outil de visualisation selon les types de communes.

Ministère de l'économie, des finances et de la relance - Produit de la CVAE réparti entre les collectivités locales en 2021 : les recettes fiscales des collectivités font preuve de résilience - Communiqué - 9 mars 2021

Le plan de relance décidé par le Gouvernement comporte un volet concernant les collectivités territoriales avec un soutien financier aux collectivités locales, à travers des mesures de garantie de recettes et de soutien à l'investissement. Malgré des critiques ci-ou-là, les données agrégées démontrent une certaine "résilience » pour reprendre les termes employés parlant d'une baisse des recettes réelles de fonctionnement limitée de $-1,4 \%$. Au final, l'épargne brute des collectivités ne diminue que de $-8,2 \%$ se retrouvant à son niveau de 2018. Concernant la CVAE, sa baisse n'est que de $-1 \%$. Mais il convient d'attirer l'attention sur le décalage des bases de cette recette fiscale ; les effets baissiers risquent de se faire ressentir sur plusieurs années.

Précisions du Conseil d'État sur les règles d'attribution des subventions locales aux cinémas (arrêt du 10 mars 2021, req. $n^{\circ} 434564$ )

Était en cause dans cette affaire, l'article L. 2251-4 du code général des collectivités territoriales selon lequel, "la commune peut attribuer des subventions à des entreprises existantes ayant pour objet l'exploitation de salles de spectacle cinématographique dans des conditions fixées par décret en Conseil d'État. Ces subventions ne peuvent être attribuées qu'aux établissements qui, quel que soit le nombre de leurs salles, réalisent en moyenne hebdomadaire moins de 7500 entrées ou qui font l'objet d'un classement art et essai dans des conditions fixées par décret». Toutefois, comme le rappelle le juge administratif, « une telle subvention ne peut, en revanche, être attribuée pour permettre la création, par une entreprise existante ayant pour objet l'exploitation de salles de spectacle cinématographique, d'un nouvel établissement de spectacle cinématographique ». Or, justement l'aide attribuée concernait un nouvel établissement. Cela explique l'annulation de l'arrêt de la Cour administrative d'appel et l'obligation qui pèse désormais sur la collectivité d'émettre un titre exécutoire aux fins de recouvrement du montant de la subvention.

\section{FINANCES SOCIALES}

\section{$\lambda$ Mesures de lutte contre la crise}

La $3^{\circ}$ conférence sociale avalise la poursuite des mesures d'aide à l'embauche des jeunes et de soutien des revenus

A l'issue de la $3^{e}$ conférence sociale tenue le 15 mars, le Premier ministre a annoncé la poursuite des mesures pour l'emploi des jeunes dans le cadre du plan « un jeune, une solution »: I'aide au recrutement d'apprentis $(8000 €)$ sera prolongée jusqu'au 31 décembre 2021, et l'aide à l'embauche de jeunes, recentrée sur les salaires inférieurs à 1,6 SMIC, jusqu'au 31 mai. Par ailleurs, la «Prime Macron » d'un montant de $1000 €$ défiscalisée et désocialisée sera recon- 
duite en 2021 pour l'ensemble des salariés, en mettant l'accent sur les travailleurs de la « deuxième ligne "; cette prime pourra être portée à $2000 €$ dans les entreprises qui auront conclu un accord d'intéressement ou auront ouvert une négociation sur la valorisation des métiers d'ici la fin de l'année. Le Premier ministre a également annoncé la mise au point d'une méthode pour la sortie de crise, mais les nouvelles mesures de la fin du mois on réintroduit la plus grande incertitude sur ce calendrier.

Le troisième confinement et l'intensification des aides aux entreprises

Suite à la mise en place d'un troisième confinement dans 16 départements (dont la région IDF) le 18 mars, le Fonds de solidarité a été une nouvelle fois ajusté en ajoutant une aide de $1500 €$ mensuels pour les entreprises perdant $20 \%$ de leur chiffres d'affaires (en fait celles fermées en fin de semaine). Mais le coût des dispositifs d'aide aux entreprises va reprendre une tout autre dimension avec l'annonce, le 31 mars, par le Président de la République $d^{\prime}$ un troisième confinement national à partir de Pâques, d'au moins 4 semaines. La fermeture des crèches et des établissements scolaires pendant 3 semaines-même si elle se recoupe de facto avec les vacances de printemps - va entrainer un recours accru à l'activité partielle, de même que la fermeture de 150000 commerces «non essentiels » va provoquer une explosion des dépenses du Fonds de solidarité. Le coût de ce troisième confinement pourrait aller bien au-delà des 0,2 points de PIB évoqués à la mi-mars pour un dispositif limité alors à 16 départements et s'élever-toutes mesures comprises - à $11 \mathrm{Md}$ f par mois selon le ministère de l'économie et des finances.

Les propositions de l'OFCE pour soutenir et relancer l'économie française

Un " policy brief " collectif de l'OFCE du 19 mars (mais qui ne prend pas en compte les conséquences du troisième confinement annoncé le 31 mars) fait un ensemble de propositions pour prolonger les différents instruments de soutien à l'économie et aux revenus. Partant de la constatation d'une chute de $8,2 \%$ du PIB en 2020, mais qui a été absorbée aux $2 / 3$ par les administrations publiques et à $1 / 3$ par les entreprises, les ménages n'ayant globalement subi aucune perte, I'OFCE anticipe tout d'abord une hausse significative du chômage en 2021, ainsi que de la pauvreté notamment chez les jeunes ; il propose de reporter la baisse du taux de prise en charge par l'État et I'UNEDIC de l'activité partielle, de différer l'entrée en vigueur de la réforme de l'assurance chômage (cf. infra) et de mettre en place 500000 contrats aidés financés à $50 \%$ par l'État (coût de ces 3 mesures: $15 \mathrm{Md}$ ) ; s'y ajouteraient diverses mesures de soutien au revenu des jeunes les plus en difficulté («NEET « : ni emploi, ni étude, ni formation), pour une dépense de 7Md€́. Pour les entreprises, qui vont se trouver très rapidement confrontées aux échéances de remboursement de prêts et de dettes fiscales et sociales, I'OFCE préconise d'adapter le Fonds de solidarité, qui a bien fonctionné pour les secteurs de l'hôtellerie-restauration et de la construction, en indemnisant mieux les pertes liées aux coûts fixes de secteurs plus capitalistiques comme l'industrie manufacturière, l'énergie, les transports et les services aux entreprises, qui représentent près de $50 \%$ de la valeur ajoutée ; ceci conduirait une enveloppe de $13 \mathrm{Md}$; par ailleurs, il propose d'aménager la recapitalisation des très grandes entreprises stratégiques comme Air France, en mobilisant, par des véhicules adaptés, le surcroît d'épargne accumulé pendant la crise. Enfin, I'OFCE suggère une expertise très approfondie du Commissariat au Plan pour évaluer les besoins de rattrapage en investissement public et en recherche développement.

\section{Situation des comptes sociaux}

\section{Le déficit 2020 de la Sécurité sociale « se limitera " à -38,9Md€}

Le ministre des comptes publics a annoncé le 9 mars, sur son compte Twitter, que le déficit de la Sécurité sociale pour 2020 serait finalement moins lourd que prévu : -38,9Md€ contre - 49Md€ attendus dans les prévisions associées à la LFSS 2021 (voir Repères de décembre). L'amélioration serait due à de meilleures recettes qu'escompté, du fait d'une moindre contraction de la masse salariale $(-5,7 \%$ contre $-8,9 \%$ prévus). Cette évolution paraît en phase avec les statistiques de I'INSEE sur l'emploi, qui font apparaître un nombre moins important de destructions d'emploi que ce qui était redouté (320 000 en 2020 et 230000 en 2021).

Le Rapport public annuel 2021 de la Cour des comptes très largement consacré à l'impact de la crise sanitaire sur les finances publiques

Le Rapport public 2021 de la Cour des comptes est très largement consacré à l'impact de la crise sanitaire. Sur les 9 chapitres de la première partie du Rapport annuel consacrés à la crise, trois concernent plus spécifiquement les po- 
litiques sociales au sens large : la réanimation et les soins critiques, le Fonds de solidarité aux entreprises, l'assurance chômage.

S'agissant de l'offre en réanimation et en soins critiques, les constats d'insuffisance très largement connus sont bien documentés par la Cour qui souligne que la gestion de crise a dû se reposer sur des déprogrammations massives sans effet de rattrapage, alors même que les capacités du secteur privé ont été peu mobilisées. La Cour relève surtout que cette situation de crise est le résultat de choix structurels-souvent implicites-qui ont été faits en matière d'offre de soins critiques, qui n'ont pas tenu compte de l'évolution démographique et du vieillissement de la population. Elle préconise d'agir plus fermement sur l'offre de soins - inégale dans les régions -, de réduire la tension sur les personnels (augmentation des effectifs de médecins réanimateurs et renforcement de la place des infirmiers) et de déterminer un nouveau modèle de financement des soins critiques à même de garantir la neutralité de la tarification à l'activité (la logique de tarification actuelle est fondée sur la maximisation du taux d'occupation des unités alors que les soins critiques nécessitent d'avoir des réserves de capacité pour faire face aux pics d'urgence).

En ce qui concerne le déploiement du Fonds de solidarité $(8,9$ Mds€ versés au 30 septembre 2020), la Cour des comptes salue la vitesse avec laquelle s'est mis en place le dispositif, la rapidité des paiements aux entreprises malgré de nombreuses évolutions réglementaires et l'accessibilité des outils pour les entreprises. Elle souligne toutefois les différences dans la mobilisation des deux volets du dispositif : volet 1 très simple (aide au revenu sous forme d'une compensation des pertes de chiffre d'affaires dans la limite de $1500 €$ mensuels); volet 2 destiné à éviter les ruptures de trésorerie les plus critiques ( versement unique entre 2000 et $5000 €)$, lui-même complété d'un volet 2 bis, ainsi que le véritable changement qui s'est opéré dans la nature du Fonds à partir de l'automne 2020 avec son ouverture aux entreprises de grande taille visées par les fermetures administratives, ce qui a fait doubler (à près de 20 Md€ à la fin 2020) l'enveloppe du Fonds. La Cour insiste sur le fait que l'élargissement de celui-ci comporte des risques (en particulier celui d'une couverture excédant le préjudice subi, du fait du cumul avec d'autres aides) et qu'il sera nécessaire de renforcer les contrôles a priori et a posteriori.
Pour la situation de l'assurance chômage, la Cour revient sur la dégradation continue du régime depuis la crise financière de 2008 et sur les différentes phases du projet de réforme du gouvernement (cf. infra); elle identifie les trois facteurs de la dégradation majeure de ses finances en 2020 : la baisse des recettes de cotisations (pour $14 \%$ de la perte due à la crise), la charge du financement de l'activité partielle, qui a pesé pour un tiers sur I'UNEDIC (56\% de la perte) et les mesures exceptionnelles en faveur des demandeurs d'emploi (prolongation de la durée d'indemnisation des allocataires arrivés en fin de droit et des droits des intermittents du spectacle, allongement de la période de référence au cours de laquelle est recherchée la durée minimale d'affiliation, suppression de la taxe forfaitaire sur les CDD d'usage) [ $30 \%$ de la perte] ; elle reprend enfin les prévisions de déficit et d'endettement très dégradées qui ont été présentées au dernier bureau de I'UNEDIC (voir Repères de février). La Cour recommande d'élargir désormais le périmètre des LFSS pour inclure I'Assurance chômage, pour tenir compte du changement du mode de financement intervenu en 2019, de rétablir I'équilibre structurel du régime, de clarifier la gouvernance entre l'État et les partenaires sociaux, et de le soulager d'une partie de son endettement qui n'est plus soutenable par lui seul. Une proposition de loi en ce sens pourrait être déposée au printemps.

\section{$\pi$ Dette sociale}

Le débat à l'Assemblée nationale sur le remboursement de la dette, parmi plusieurs contributions

Le débat sur le remboursement de la dette bat son plein. Intervenant le 22 mars à l'Assemblée nationale, les ministres des finances et des comptes publics ont esquissé une stratégie de remboursement de la dette COVID (estimée à $215 \mathrm{Md}$, dont $140 \mathrm{Md}$ f pour l'État et $75 \mathrm{Md}$ d pour la Sécurité sociale). Bruno Le Maire a présenté les " 4 piliers de la "sagesse financière": le retour de la croissance, la maîtrise de la dépense publique avec l'instauration d'une règle pluriannuelle de dépense, l'affectation à la dette de l'État d'une part de la ressource fiscale générée par la croissance - sans créer d'impôt nouveau mais sans exclure une contribution accrue des entreprises (la dette sociale étant elle déjà traitée en partie par la prolongation de la CADES actée à l'été dernier- voir Repères d'août), enfin la mise en œuvre de réformes de structure (et en particulier la réforme des re- 
traites). Olivier Dussopt a précisé la situation de la dette sociale, estimée à $75 \mathrm{Md}$ pour les années 2020 et antérieures, ce qui laisserait une certaine marge par rapport à l'enveloppe de 136 MdE transférée à la CADES. Plusieurs contributions importantes sont intervenues durant cette période : le 18 mars, le rapport de la Commission sur l'Avenir des finances publiques », présidée par Jean Arthuis, n' a pas retenu, à proprement parler, l'option de cantonnement de la dette, mais a préconisé une stratégie de désendettement fondée sur une maîtrise des dépenses en deçà de l'évolution des recettes ; cela au prix d'une réforme fondamentale de la gouvernance des finances publiques axée sur 3 piliers : I'instauration d'une norme de dépense pluriannuelle, en conservant un niveau «plancher » pour les « dépenses d'avenir », l'installation d'une "vigie budgétaire » indépendante pour avoir une approche de long terme sur la soutenabilité, l'approfondissement du débat parlementaire en privilégiant l'évaluation. Enfin le 24 mars, le Haut conseil pour le financement de la protection sociale a remis une note d'étape : il réaffirme la nécessité d'un pilotage de la Sécurité sociale par les soldes pour assurer le retour à l'équilibre, en prenant pour exemple les régimes de retraite complémentaire, mais sans renoncer à la fixation d'objectifs de dépenses comme l'ONDAM; le HCFiPS préconise, pour sa part, de prioriser le retour à l'équilibre des comptes courants, plutôt que de rembourser rapidement la dette, critiquant ainsi ipso facto ce qui a été fait pour la CADES ; il est favorable à une rénovation du cadre pluriannuel, en recherchant une meilleure articulation avec les politiques de santé publique ; enfin, il apporte un éclairage particulier sur la situation de l'Assurance maladie, parce que la dynamique des dépenses de cette branche va toujours dépasser l'évolution de ses recettes fondée sur la croissance, et son retour à l'équilibre en 2030-2040 supposerait une compression de I'ONDAM qui apparaît hors de portée.

\section{त Dépenses de santé/Hôpital/ Assurance maladie}

Le Premier ministre annonce un plan de 19 Md€ pour l'investissement en santé

En déplacement à Cosne sur Loire le 9 mars, Jean Castex a dévoilé le volet « investissement en santé » du Ségur de la Santé, qui fait suite au mesures en faveur des personnels arrêtées en juillet 2020 (voir Repères de juillet). Ce plan de 19 Md€ s'étalera sur 10 ans : 6,5 Md€ seront consacrés à l'allègement de la dette des hôpi- taux, ce qui devrait leur permettre d'améliorer leur capacité d'autofinancement, 1,5 Md€ à l'extension des capacités des EHPAD, 2 Md€ à la création de de l'Espace numérique en santé, 0,3 Md€ à l'amélioration de l'offre de soins pour les personnes en situation de handicap, et $9 \mathrm{Md} €$ pour le financement de nouveaux investissements en établissements de santé (dont 6,5 Md€ pour des projets de transformation de I'offre de soins). La mise en œuvre du plan d'investissement reposera en priorité sur les ARS, et, selon le Premier Ministre, la méthode se veut en rupture avec celle suivie depuis une décennie, en reposant sur les principes suivants: déconcentration (seuls les projets supérieurs à 150M€ feront l'objet d'une approbation au niveau national), recours à une expertise technique forte fondée sur l'accompagnement plutôt que sur le contrôle, territorialisation des problématiques d'offre de soins, transparence dans l'évaluation médicale et soignante des projets.

\section{$\pi$ Retraites}

Le poids toujours croissant des retraites dans la dépense publique

Une étude que vient de publier le site FIPECO fait apparaître le poids croissant des dépenses de retraite dans la dépense publique en France. En 2019, les dépenses publiques représentaient en moyenne 55,6\% du PIB, contre $47 \%$ en moyenne dans la zone euro, avec une augmentation continue en France depuis 2001. Les dépenses sociales représentaient 31,9\% du PIB en France contre $27 \%$ en moyenne dans la zone euro, la France étant en avance dans tous les domaines, mais avec un écart particulièrement important pour les dépenses de retraite (14,6\% vs 12,6\%). Selon l'étude, cet écart s'explique par le fait que les régimes de retraite complémentaires ont en France un caractère obligatoire (ce qui les fait classer en dépenses des administrations publiques), que les niveaux moyens de pensions y sont plus élevés et que l'espérance de vie à la retraite est plus longue. Si on considère la dynamique de la dépense, ce sont encore les dépenses de pensions qui ont tiré vers le haut depuis 2001, avec $+2,8$ points en France contre $+1,5$ points dans la zone euro.

Les régimes de retraite complémentaires retombent dans le rouge

Selon les informations parues dans Les Echos, I'AGIRC-ARRCO a enregistré un déficit de 4,8 Md€ en 2020 après un excédent de 1 Md€ en 2019. C'est la conséquence de la contraction de la masse salariale qui a entrainé une 
baisse des rentrées de cotisations, cependant inférieure à ce qui était redouté, ainsi que de la politique de délais et de reports de paiement, tout en assurant le maintien des droits des salariés en activité partielle. Les réserves financières ont diminué de près de $4 \mathrm{Md}$, en revenant à un montant de 61,7 Md€ ; ce « matelas » devrait permettre d'assurer le volant minimal requis de 6 mois de prestations jusqu'en 2026, ce qui ne conduit pas le régime à envisager de nouvelles mesures de redressement à ce stade.

\section{$\lambda$ Formation professionnelle}

Les partenaires sociaux reprennent les discussions sur la formation professionnelle

Après la grande réforme de la formation professionnelle intervenue en 2018, le patronat et les syndicats (sauf la CGT, ce qui apparaît comme une rupture dans la pratique de cette centrale) ont arrêté le 23 mars un calendrier de discussions jusqu'en juin pour faire le bilan de la loi, et se pencher notamment sur les besoins en compétences et qualifications, l'accompagnement des salariés au cours de leur parcours professionnel et les transitions professionnelles.

\section{त Assurance chômage}

La réforme de l'assurance chômage entrera en vigueur le $1^{\mathrm{er}}$ juillet sous conditions

La ministre du travail a présenté le 2 mars aux partenaires sociaux les arbitrages du gouvernement sur la réforme de l'assurance chômage. S'agissant de l'éligibilité à l'indemnisation, le nombre de mois de cotisations nécessaire va passer à 6 mois sur les 24 derniers mois (contre respectivement 4 et 18 actuellement), sous réserve d'une amélioration du marché de l'emploi conditionnée par deux indicateurs (la baisse de 130000 du nombre d'inscrits en catégorie à Pôle Emploi sur 6 mois glissants et le maintien au- delà de 2,7 millions du nombre de déclarations d'embauche sur 4 mois). La détermination du salaire journalier de référence qui sert au calcul de l'indemnisation a été un peu assouplie par rapport aux modalités de la réforme qui ont présentées en 2019 pour éviter une chute trop brutale des allocations. Ce sont ces deux dispositions-destinées à lutter contre la « permittence » qui rencontrent le plus d'hostilité de la part des syndicats, et de scepticisme de la part de certains économistes. La dégressivité des allocations pour les cadres gagnant plus de $4500 €$ bruts mensuels est maintenue, sous réserve que les clauses de retour à meilleure fortune du marché de l'emploi visées ci-dessus soient vérifiées. Enfin le "bonus-malus» pour I'usage de contrats courts est maintenu, mais avec un sursis pour les secteurs les plus touchés par la crise comme l'hôtellerie-restauration. Ces nouvelles modalités, qui ne pourront générer qu'un montant d'économies sensiblement en retrait par rapport à ce qui était prévu il y a 2 ans devraient prendre effet à compter du 1 er juillet 2021 si les conditions sont réunies. Selon I'UNEDIC, la réforme ferait baisser les indemnisations de 1,1 million d'allocataires d'environ $17 \%$ en moyenne et pourrait procurer une économie de 2,3 Md€ en régime de croisière. Le décret entérinant les nouvelles dispositions est paru au JO du 31 mars.

\section{त Solidarité/lutte contre la pauvreté/ Revenu universel}

La forte augmentation du nombre d'allocataires au RSA en 2020

Selon les statistiques provisoires de la CNAF, le nombre d'allocataires du RSA devait être de 2,06 millions en décembre 2020, soit une progression de 7,5\% sur un an. Cette forte augmentation fait suite à une longue période de stabilité qui avait duré de janvier 2017 à février 2020, et est due plus à des « rechutes » d'anciens allocataires qu'à l'entrée de nouveaux bénéficiaires, ainsi qu'à la réduction du nombre de sorties. La prime d'activité a peu progressé, avec 4,58 millions de foyers bénéficiaires en décembre 2020 , soit une augmentation de 2,1\% en un an.

\section{FISCALITÉ ET PROCÉDURE FISCALE}

\section{$\pi$ Procédure fiscale}

La constitutionnalité de l'amende pour défaut de facturation en question

Le Conseil d'État vient de transmettre au Conseil constitutionnel une QPC portant sur les dispositions de l'article 1737, I-3 du CGI (CE, 24 février 2021, Société KF3 Plus, Req. n443476) qui sanctionne les professionnels ne respectant pas l'obligation de délivrance d'une facture à leurs clients d'une amende égale à $50 \%$ du montant de la transaction, le client étant solidairement tenu au paiement de cette amende. Cette amende est-elle susceptible de porter atteinte au principe de nécessité des peines? A suivre... 


\section{$\lambda$ Impôt sur les sociétés}

Le changement d'activité et la perte du droit au report des déficits

Si le report illimité des déficits demeure un principe général du droit fiscal, le Conseil d'État vient de rappeler qu'il comporte des exceptions, notamment en cas de changement d'objet social ou d'activité réelle de la société (art. 209-I et 221-5 du CGI). La mise en œuvre du droit au report déficitaire est notamment subordonnée à la condition que la société qui s'en prévaut n'ait pas subi, dans son activité réelle, de transformations telles qu'elle ne serait plus, en réalité, la même. En l'espèce, en estimant que l'activité de la société requérante avait changé au 1 er janvier de l'année, le Conseil d'État a considéré que la CAA de Versailles n'avait pas inexactement qualifié les faits qui lui étaient soumis en considérant que la société requérante avait perdu tout droit au report de ses déficits antérieurs conformément aux dispositions de l'article 209 du CGI (CE, 9e ch., 8 mars 2021, Société Afli, Req. n430674).

\section{$\pi$ Impôt sur le capital}

La proposition de loi en vue d'alléger la fiscalité sur la transmission du capital : une évaluation?

Alors que le Gouvernement veut faire un geste fiscal en faveur des donations, plusieurs députés de l'opposition ont déposé une proposition de loi visant à alléger la fiscalité applicable aux successions et aux donations (A.N., proposition $n^{\circ} 3962,9$ mars 2021) : allègement des droits de succession (art. $777 \mathrm{du}$ CGI) afin de ramener la tranche marginale en ligne directe à $30 \%$ au lieu de $45 \%$, avec une tranche principale à $15 \%$ et restaurer l'abattement de $200000 €$ au lieu de $100000 €$ à ce jour ; suppression du droit de partage ; exonération de droits de mutation à titre gratuit pour les donations entre époux. Le chiffrage du coût de cette proposition lui éviterait de rester lettre morte. II est grand temps que le nouvel outil de simulation Leximpact dont disposent les députés de la commission des finances pour chiffrer l'impact de leurs propositions et amendements, afin de renforcer les moyens d'évaluation et de contrôle du Parlement, ne soit plus seulement limité à l'impôt sur le revenu. D'ailleurs, selon le député Saint-Martin, rapporteur général du budget à l'Assemblée nationale : « il faut rendre les donations plus attractives pour faire sortir l'épargne » (interview dans Les Échos, 10 mars 2021). Un travail parlementaire préalable et autonome d'évaluation des propositions de loi en matière fiscale, en contrepoids des projets du Gouvernement, est donc urgent.

Les ISF : le feuilleton judiciaire "Finaréa " devant la Cour de cassation (2)

L'épilogue du feuilleton judiciaire (v. Repères, janv. 2021) dans l'affaire Finaréa? Dernièrement, la Cour de cassation a donné raison à l'administration fiscale sur la valeur des attestations fiscales et le concept de "holding animatrice ". Sur ce dernier point, la Cour de cassation a considéré que tant que la holding n'a pas pris de participation dans une PME, le souscripteur au capital de cette holding ne peut profiter d'une réduction d'ISF, en dépit de la mécanique d'amorçage ou de démarrage propre à ce type d'investissement. Cette décision a posé la question plus large de la valeur des attestations fiscales remises par des tiers à des contribuables, en vue d'une réduction d'impôt, d'un crédit d'impôt ou d'une déduction de revenu imposable. Est-ce que cette attestation offre un droit au contribuable de bonne foi à ne pas être redressé ? Contrairement à la majorité des tribunaux qui se sont prononcés depuis dix ans sur ce dossier tentaculaire, la Cour de cassation a répondu «non ». De nombreux observateurs y ont vu un facteur d'insécurité juridique pour les contribuables (F. Perrotin, LPA, 29 mar. 2021, n 160, p. 4 ; D. Villemot, «ISF et égalité des armes, I'affaire Finarea ", Revue Droit fiscal, mars 2021, 153). Cela a même fait l'objet d'une mise au point de Bercy sur les attestations fiscales une semaine après les six arrêts rendus par la Cour de cassation ( Cass. com., 3 mars 2021, Finaréa, Pourvois n¹9-22.397, 18-15.826, 19-21.161, 20-11.838, 20-11.839, 20-11.840) dont cinq prévoient un renvoi de l'affaire en cour d'appel. A suivre...

\section{$\lambda$ Impôt sur le revenu}

Télétravail : les allocations pour frais professionnels exonérées d'impôt sur le revenu

Les allocations pour frais professionnels versées par les employeurs au titre du télétravail à domicile en 2020 seront exonérées d'impôt sur le revenu selon le ministre de l'économie et des finances et le ministre délégué chargé des comptes publics.

\section{入 Fiscalité environnementale}

La fin de l'avantage fiscal sur les biocarburants à partir d'huile de palme 
Le Conseil d'État a considéré que tous les produits à base d'huile de palme devaient être exclus de la liste des biocarburants bénéficiant $d^{\prime}$ un avantage fiscal $\left(C E, 9^{e}\right.$ et $10^{e}$ ch. r., 24 février 2021, Associations Canopée, Les Amis de la Terre et Greenpeace France, Req. $n^{\circ}$ 437277) à la demande de plusieurs associations requérantes lui demandant l'annulation pour excès de pouvoir de la note d'information des Douanes en date du 19 décembre 2019 intégrant les biocarburants produits à base d'huile de palme dans la liste des produits bénéficiant de la réduction de la taxe incitative relative à I'incorporation de biocarburants (TIRIB - art. 266 quindecies du code des douanes). Le Conseil d'État relève qu'aux termes du dernier alinéa du B du V de l'article 266 quindecies du code des douanes, "ne sont pas considérés comme des biocarburants les produits à base d'huile de palme ». Le Conseil constitutionnel avait d'ailleurs considéré que le législateur (art. 192 L. n²018-1317 du 28 déc. 2018 de finances pour 2019) avait pu, sans méconnaître la Constitution, exclure l'huile de palme d'un régime fiscal favorable prévu pour les biocarburants (Cons. const., décis. n²019-808 QPC, 11 oct. 2019, Société Total raffinage France). Le Conseil d'État a donc annulé la note d'information de l'administration des douanes ayant méconnu les dispositions législatives dont elle entendait préciser l'application.

\section{Le retour de l'écotaxe poids-lourds?}

Le législateur a créé, à titre expérimental, une écotaxe poids lourds régionale pour les véhicules de plus de 3,5 tonnes sur le territoire des départements d'Alsace, de Moselle, de Meurthe-et-Moselle et des Vosges afin de lutter contre le report sur l'axe rhénan de camions étrangers venus en France pour échapper au système de tarification mis en place en Allemagne depuis 2005 (v. Actes du séminaire de la Société Française des Finances Publiques à Lille sur la fiscalité environnementale, Revue GFP, $n^{\circ}$ 2021/2). Dernièrement, les députés de la commission spéciale sur le projet de loi Climat ont donné leur accord à l'instauration de contributions régionales sur les poids lourds sur la base du volontariat des collectivités concernées. Un vote qui ouvre la voie à un retour d'une écotaxe enterrée sous la législature précédente à la suite de la fronde des «bonnets rouges ».

\section{Management PUBlic}

\section{$\lambda$ Services déconcentrés}

Le renforcement du télétravail dans les services déconcentrés

Le gouvernement demande aux préfets d'intensifier le télétravail dans les services déconcentrés. Dans une instruction du 18 mars 2021, le ministre de l'Intérieur, la ministre du travail et la ministre de la transformation et de la fonction publiques invitent les représentants de l'État à "faire remonter", avant le 22 mars 2021, toutes les actions envisagées pour remédier à un niveau insuffisant de travail en distanciel, en particulier dans 8 départements identifiés.

\section{$\pi$ Collectivités territoriales}

La simplification des expérimentations locales

Les députés ont adopté sans modification, le 16 mars 2021, le projet de loi organique de simplification des expérimentations approuvé par les sénateurs au mois de novembre 2020. Par ce vote en termes conformes, le texte est donc considéré comme définitivement adopté, sans qu'il ne soit besoin d'avoir recours à une commission mixte paritaire.

\section{Les intercommunalités et la relance}

Le Premier ministre et le président de l'Assemblée des communautés de France (ADCF), ont signé le 22 mars 2021 un accord de partenariat avec l'Assemblée des communautés de France (ADCF) concernant la mise en œuvre des contrats de relance et de transition écologique (CRTE). Selon le communiqué de presse publié, " les CRTE ont été conçus pour contribuer à la mise en œuvre de plusieurs mesures du plan France relance au plan local et pour accompagner les grandes transitions économiques, écologiques et de cohésion à l'œuvre dans tous les territoires. Ils ont vocation à demeurer évolutifs et s'inscrire dans la durée du mandat municipal 2020/2026».

\section{$\lambda$ Relations avec les usagers}

La nouvelle carte nationale d'identité électronique

Le 16 mars 2021, la ministre déléguée à la citoyenneté a annoncé la mise en œuvre de la nouvelle carte d'identité électronique dans I'Oise en vue d'une généralisation du dispositif à l'ensemble du territoire au mois d'août 2021.

Le décret n² 2021-279 du 13 mars 2021 précise que la carte comportera "l'image numérisée de la photographie ainsi que (...) l'image numérisée des empreintes digitales de deux doigts ». Cette évolution s'inscrit dans le prolongement 
du règlement (UE) n²019/1157 du Parlement et du Conseil du 20 juin 2019 qui impose le renouvellement des cartes d'identité pour y implanter une puce électronique (dite "ICAO») destinée à héberger les données biométriques de chaque détenteur.

Les concours et les examens durant la crise du Covid-19

La direction générale de l'administration et de la fonction publique (DGAFP) vient d'actualiser ses recommandations relatives au déroulement des concours et examens dans le contexte de la situation épidémique liée au Covid-19. On retiendra notamment que :

- un concours ou un examen de la fonction publique doit être regardé comme un rassemblement, réunion ou activité à caractère professionnel ;

- les trajets à destination ou en provenance du lieu d'organisation d'un examen ou d'un concours font partie des déplacements dont les motifs dérogent aux restrictions de circulation entre 19 heures et 6 heures du matin ;

- quel que soit le moyen de transport concerné pour les candidats aux concours et examens, la convocation vaut justificatif de déplacement.

\section{$\pi$ Gestion publique}

Le Rapport public annuel 2021 de la Cour des comptes

Si la Cour a choisi de consacrer la première partie de son rapport public annuel (RPA) 2021 à différents thèmes directement liés à cette crise (cf. partie Finances sociales), la seconde partie du RPA propose, plus classiquement, un échantillon représentatif des travaux, tant de la Cour que des chambres régionales et territoriales des comptes.

Le remboursement de frais des élus en situation de handicap

Le décret $n^{\circ}$ 2021-258 du 9 mars 2021 fixe les conditions de remboursement des frais spécifiques de déplacement, d'accompagnement et d'aide technique engagés par les élus locaux en situation de handicap dans les mêmes conditions que les élus municipaux, départementaux ou régionaux. Ce texte a été pris en application de la loi n²019-1461 du 27 décembre 2019 relative à l'engagement dans la vie locale et à la proximité de l'action publique (dite « loi Engagement et Proximité »).
Le recrutement des conseillers numériques

Le plan de recrutement des 4000 « conseillers numériques France services » qui s'inscrit dans le cadre du plan de relance est désormais ouvert aux structures privées, comme en précise le texte de l'appel à manifestation d'intérêt qui a été rendu public. Les associations et les acteurs de l'économie sociale et solidaire (coopératives, mutuelles, fondations) peuvent désormais manifester leur intention de "porter les contrats de travail de ces conseillers en contrepartie d'une subvention de l'État permettant la prise en charge partielle de leur formation et de leur rémunération ».

\section{$\pi$ Fonction publique}

La place croissante des contractuels dans la fonction publique en 2019

Selon une analyse de l'INSEE publiée au mois de mars 2021, 5,66 millions de salariés (1 salarié sur 5) travaillaient dans la fonction publique fin 2019, soit 18300 de plus que fin 2018 (+ 0,3\%). Sur la même période, on constate que le nombre de fonctionnaires poursuit sa baisse $(-0,3 \%)$ tandis que le nombre de contractuels augmente de 5,2 \% (55 600 personnes dont 25400 d'emplois aidés). Au total, les contractuels représentaient 19,9\% des emplois à la fin de l'année 2019.

\section{L'évolution du statut des contractuels}

Le ministère de la transformation et de la fonction publiques a décidé d'initier la modification du décret 17 janvier 1986 fixant les dispositions générales applicables aux agents contractuels de l'État. S'inscrivant dans le prolongement de la loi n²019-828 du 6 août 2019 de transformation de la fonction publique, cette démarche est destinée à rendre les questions statutaires plus lisibles, à adapter des dispositions devenues obsolètes et à harmoniser les droits des personnels contractuels avec ceux des fonctionnaires.

\section{$\pi$ Ressources documentaires}

\section{L'Atlas des départements français}

L'Agence nationale de la cohésion des territoires (ANCT) a publié au mois de février 2021 un Atlas des départements français. Ce document synthétique, composé de cartes et de graphiques, décrit la situation des départements français métropolitains et ultramarins. 
Le baromètre de l'égalité femmeshommes

À l'occasion de la Journée internationale des droits des femmes, le 8 mars 2021, le ministère de la justice a publié un baromètre de l'égalité femmes-hommes. On y trouve, notamment, des données relatives à la féminisation des postes à la Chancellerie.

\section{EUROPE}

\section{$\pi$ Politique européenne}

Aides d'État : recours de la Commission contre le Royaume-Uni

Le 15 mars, alors qu'elle accueille actuellement avec bienveillance les aides d'État permettant de répondre à la crise sanitaire, la Commission européenne a saisi la Cour de justice d'un recours contre le Royaume-Uni au motif qu'il n'a pas récupéré intégralement des aides illégales d'un montant maximal d'environ $100 \mathrm{M} €$ octroyées dans le cadre d'un régime d'exonération fiscale à Gibraltar entre 2011 et 2013. Londres avait jusqu'au 23 avril 2019 pour récupérer toutes ces aides illégales. Le « Brexit» n'est pas soldé.

Déclaration de l'Eurogroupe en faveur d'une réponse budgétaire coordonnée à la crise pandémique

Le 15 mars, l'Eurogroupe a réuni en vidéoconférence les ministres des finances qui ont fait le point sur les mesures de soutien budgétaire, en mettant l'accent sur la stratégie et l'orientation budgétaires dans la zone euro. Ils ont adopté une déclaration en faveur d'une réponse budgétaire coordonnée à la crise sanitaire pour favoriser la reprise économique.

\section{$\lambda$ Budget européen}

Les députés prêts à poursuivre la Commission en justice pour protéger le budget de I'UE

Le 25 mars 2021, une majorité 529 députés européens (148 contre et 10 abstentions) a adopté une résolution pour rappeler que le non-respect par les pays de l'UE de l'état de droit peut porter atteinte à l'intégrité du budget de l'Union. Ils informent la Commission que si elle ne respecte pas les obligations qui lui incombent au titre du règlement juridiquement contraignant sur la conditionnalité (Règlement $n^{\circ}$ 2020/2092 du 16 décembre 2020) et ne prend pas toutes les mesures appropriées pour défendre les valeurs et les intérêts financiers de
I'UE, le Parlement « considérera ce fait comme étant constitutif d'une carence » et poursuivra la Commission en justice en vertu de l'article 265 du traité FUE. Sur ce sujet, mais sans rapport avec la conditionnalité des fonds européens, la Commission a formé le 31 mars un recours contre la Pologne devant la Cour de justice de I'UE afin de protéger l'indépendance des juges polonais.

\section{Fiscalité}

Proposition de renouvèlement du régime de l'octroi de mer

Le 3 mars 2021, la Commission a proposé de renouveler le régime fiscal spécial des régions ultrapériphériques françaises de I'UE pour la période 2022-2027.

Nouvelle directive fiscale relative à la coopération administrative

Le 22 mars 2021, le Conseil a modifié la directive 2011/16/UE relative à la coopération administrative dans le domaine fiscal. Les nouvelles règles adoptées visent à renforcer la coopération administrative et à inclure les ventes réalisées sur des plateformes numériques. Elles imposent aux opérateurs de ces dernières de déclarer les revenus perçus par les vendeurs sur leurs plateformes et aux États membres d'échanger automatiquement ces informations. Elles s'appliqueront à partir du 1er janvier 2023. Elles permettront aux autorités fiscales nationales de détecter les revenus perçus par l'intermédiaire de plateformes numériques et de déterminer les obligations fiscales en résultant. La déclaration se fera dans un seul État membre conformément à un cadre commun de I'UE.

Résolution du Parlement européen en faveur d'une taxe carbone sur les importations

Mercredi 10 mars, le Parlement a adopté une résolution sur un mécanisme européen d'ajustement des émissions de carbone aux frontières compatible avec l'OMC par 444 voix pour, 70 contre et 181 abstentions. Les députés soutiennent l'imposition d'une tarification du carbone sur certains biens importés depuis des pays tiers, si ces pays ne sont pas assez ambitieux en matière de changement climatique. Selon ce texte, cela " créerait des conditions équitables au niveau mondial et permettrait $d$ 'inciter les industries européennes et non-européennes à décarboner », conformément aux objectifs souscrits par l'Union dans le cadre de l'accord de Paris. 


\section{入 États-membres}

La Hongrie et la Pologne saisissent la CJUE sur le conditionnement des aides

La Hongrie et la Pologne ont annoncé, jeudi 11 mars 2021, avoir saisi la Cour de justice de I'Union européenne (CJUE) d'une plainte contre le mécanisme conditionnant les versements de fonds européens au respect des règles de l'état de droit. Ce Règlement n²020/2092 avait été adopté lors d'un Conseil européen du 16 décembre 2020 selon la procédure normale, c'està-dire de vote à la majorité, que ces deux pays avaient essayé de bloquer en s'opposant momentanément à l'adoption du cadre financier pluriannuel et au plan de relance, exigeant, eux, I'unanimité des États membres. Selon eux, le Règlement serait illégal, car, pour la Pologne, "I'Union n'a pas la compétence pour définir la notion d'état de droit ", notion inscrite aux valeurs fondatrices de l'article 2 du Traité sur I'Union européenne.

Le Tribunal constitutionnel allemand suspend la ratification du plan de relance

Par une décision rendue vendredi 26 mars, le Tribunal constitutionnel allemand a ordonné la suspension du processus de ratification du fonds de relance européen en raison d'un recours contre le mécanisme utilisé, fondé sur une dette commune européenne. Les deux chambres du Parlement national venaient tout juste d'approuver le texte de loi. Le recours empêche la signature du chef de l'État allemand, Frank-Walter Steinmeier, indispensable pour sa ratification, qui devra attendre plusieurs semaines.

\section{INTERNATIONAL}

\section{ス États}

États-Unis : le plan de relance est adopté

Le 10 mars 2021, le président Biden a obtenu du Congrès l'adoption de son plan de relance économique de 1900 milliards de dollars. Le Sénat I'avait adopté le samedi 6 mars (par 50 voix contre 49) et la Chambre des représentants I'a suivi par 220 voix contre 211 . Ce montant « colossal » équivaut au PIB de I'Italie. Le plan comprend notamment des chèques d'aides directes allant jusqu'à 1400 dollars par individu et personne à charge, pour un montant global de quelque 400 milliards de dollars.

\section{त Relations multilatérales}

UE-États-Unis : suspension du conflit aérien

Le 5 mars 2021, L'Union européenne et les États-Unis ont convenus de suspendre, pour une période de quatre mois, toutes les mesures tarifaires de rétorsion imposées sur les exportations européennes et américaines dans le cadre des différends liés à Airbus et Boeing.

\section{$\pi$ Monnaies}

Réunion des banques centrales consacrée aux monnaies numériques

Du 22 au 25 mars 2021, les banques centrales se sont réunies virtuellement sous l'égide de la Banque des règlements internationaux pour discuter des monnaies numériques, notamment pour répondre notamment aux projets comme la «libra » de Facebook, rebaptisée " Diem». Selon la BRI, $86 \%$ des établissements monétaires nationaux réfléchissent à un projet de "monnaie numérique de banque centrale» (MNBC) reposant notamment sur la blockchain (" chaîne de blocs »), une technologie permettant de crypter, de répertorier et de sécuriser les transactions.

Aurélien BAUDU (Fiscalité et procédure fiscale),

Fabrice BIN (Europe, international),

Jean-François CALMETTE (budget de l'État et des opérateurs),

Matthieu HOUSER (finances locales), Michel LE CLAINCHE (comptabilité publique, management public, coordination), Yves TERRASSE (finances sociales), Robin DEGRON (coordination).

Retrouvez sur www.rgfp.fr :

- I'intégralité des REPÈRES d'actualité des finances et de la gestion publiques depuis 2015

- le 15 du mois, les REPÈRES d'actualité du mois précédent. 